NISTIR 7866

\title{
Next Generation Packaging, Kitting, and Palletizing: Can one robot do it all?
}

Raj Madhavan Stephen Balakirsky

Craig Schlenoff

http://dx.doi.org/10.6028/NIST.IR.7866 


\title{
Next Generation Packaging, Kitting, and Palletizing: Can one robot do it all?
}

\author{
Raj Madhavan \\ Stephen Balakirsky \\ Craig Schlenoff \\ Intelligent Systems Division \\ Engineering Laboratory \\ National Institute of Standards and Technology
}

http://dx.doi.org/10.6028/NIST.IR.7866

June 2012

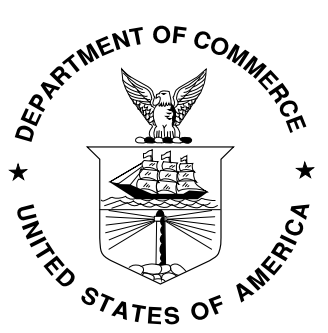

U.S. Department of Commerce Rebecca Blank, Acting Secretary 


\title{
Next Generation Packaging, Kitting, and Palletizing: Can one robot do it all?
}

\author{
R. Madhavan, S. Balakirsky, and C. Schlenoff
}

\section{Executive Summary}

In the material handling community, three disparate subgroups exist in the areas of kitting, packaging, and palletizing. Currently, these operations are performed by a mix of automated and manual systems with very limited market penetration of automated systems into small- and midsized companies. Lack of automation is due to difficulties in using today's generation of robots for tasks that include requirements such as small lot sizes and product variability. In addition, infrastructure requirements and high levels of required expertise make them prohibitively expensive. A major contributing factor is that current manufacturing robots have little or no understanding of the world around them and no capability to dynamically change their actions if the environment or task changes. This restricts them to operate in highly constrained environments and makes it difficult to change from one task to another.

A seminar titled "Next Generation Packaging, Kitting, and Palletizing: Can one robot do it all?” was held on February $9^{\text {th }} 2012$ at the Material Handling Industry of America (MHIA) MODEX show in Atlanta, GA. The seminar primarily focused on whether systems that are capable of modifying their behavior by generating and adapting plans will overcome the aforementioned limitations and if these systems will require detailed knowledge of and a consistent representation for both the manufacturing environment and the plans. The seminar also explored ways to exploit the significant similarities in packaging, kitting, and palletizing in the way their requirements are represented, and in how their performance is evaluated.

The seminar was attended by over twenty end-users, robot vendors, system integrators, and researchers. It consisted of six talks by speakers from industry and the National Institute of Standards and Technology (NIST), followed by a general discussion. The group discussed how to collaborate, with industry participants from all sectors soliciting NIST's leadership in developing a data representation to describe product handling for packaging, kitting, and palletizing. The participants recognized that there is a large overlap in the technologies needed for packaging, kitting, and palletizing, and decided to set up an informal working group that will use mailing lists to identify issues to address jointly. A subset of the participants also agreed to act as an industry advisory panel for a NIST project that is addressing these issues.

This document ${ }^{1}$ reports on the issues discussed at the workshop and next steps.

\footnotetext{
${ }^{1}$ Certain commercial equipment, instruments, or materials are identified in this document. Such identification does not imply recommendation or endorsement by the National Institute of Standards and Technology nor does it imply that the products identified are necessarily the best available for the purpose. Next Generation Packaging, Kitting, and Palletizing: Can one robot do it all?
} 


\section{Introduction}

Researchers from the Intelligent Systems Division (ISD) of the National Institute of Standards and Technology (NIST) brought together vendors, integrators, and end-users to participate in a half-day seminar on February $9^{\text {th }} 2012$ at the MODEX show held in Atlanta. The primary focus of the seminar was to explore material handling with respect to kitting, packaging, and palletizing in order to find areas of common interest where resources may be combined to provide impact to the overall industry.

The half-day seminar consisted of an introductory talk by NIST followed by talks from three vendors, one integrator, and one end-user with over twenty attendees representing various communities from industry, academia, and government. An open discussion concluded the seminar during which the participants and the speakers discussed existing gaps, easily achievable short-term goals, long-term wish lists, and the steps required to close the existing gap between these communities. A subset of the participants also agreed to act as an industry advisory panel for a NIST project on Intelligent Planning and Modeling for Autonomous Systems ${ }^{2}$ that is addressing these issues.

\section{Goals and Motivation}

Currently, material handling infrastructure associated with manufacturing and assembly operations is a mix of automated and manual systems with very limited market penetration of automated systems into small- and mid-sized companies. Lack of automation is due to difficulties in using today's generation of robots for tasks that include requirements such as small lot sizes and product variability. In addition, infrastructure requirements and high levels of required expertise make them prohibitively expensive. A major contributing factor is that current manufacturing robots have little or no understanding of the world around them and no capability to dynamically change their actions if the environment or task changes. This restricts them to operate in highly constrained environments and makes it difficult to change from one task to another. NIST believes that systems that are capable of modifying their behavior by generating and adapting plans will overcome these limitations and that these systems will require detailed knowledge of and a consistent representation for both the manufacturing environment and the plans.

In the material handling community, three disparate subgroups exist in the areas of kitting, packaging, and palletizing. There are, however, significant similarities in these tasks, in the way their requirements are represented, and in how their performance is evaluated. This seminar explored these similarities and looked for ways in which common representations and automated planning systems can be developed to enhance performance and simplify setup and execution. The desired long-term end results are next generation material handling systems and the measurement science and standards for planning and modeling. The resulting systems will be flexible enough for use in a wide range of applications.

\footnotetext{
${ }^{2}$ http://www.nist.gov/el/isd/ps/intellplanmodautosys.cfm Next Generation Packaging, Kitting, and Palletizing: Can one robot do it all?
} 


\section{Summary of the Talks}

The following is a summary of the key points from the talks delivered by the invited speakers:

- Title: Intelligent Planning and Modeling for Autonomous Systems

Stephen Balakirsky of NIST provided an overview of a recently embarked project within NIST that is developing measurement science and standards to enable advancements in the autonomous decision-making of robots through application to specific scenarios relevant to manufacturing. The ability to create and execute plans in real-world scenarios is what separates a machine-tool from an intelligent manufacturing system. Plans enable a robot to change its actions to deal with uncertainty in its environment and to rapidly switch to new tasks. These plans are based on models of the current environment, predictions about the future, and a priori knowledge of causal relationships between current actions and results. He argued that the breadth and usability of knowledge in these models is one of the main factors that constrains the flexibility and performance of manufacturing planning systems. There is currently no accepted standardized way to represent this knowledge, to reason with this knowledge, or to measure the performance of these systems.

- Title: Meeting the Challenges of Mixed Case Palletizing in Production Larry Sweet of Symbotic (a new supply chain automation division of end-user C\&S Wholesale Grocers) shared his company's experience in case picking and palletizing. In the conventional case picking operations at C\&S, they employ voice-pick systems that allows each employee to handle close to 150 cases an hour, with warehouses handling approximately a million cases per week or more. He remarked that in a typical grocery supply chain only one-sixth of the cost is associated with the raw material and production of the product, most of which is largely automated already. The remainder is largely labor, workers comp, and transportation, which provides the greatest opportunity for automation. As for the use of robots in these operations, a layer picking robot breaks down single Stock Keeping Unit (SKU) or layer rainbow pallets, and using appropriate procedures, the cases are then stored and retrieved in the right sequence for building a mixed pallet. He gave examples from the beverage and grocery industries of the challenges involved in building, handling, and grouping pallets due to the fact that even within the same SKU there may be products of different dimensions, variations in height, "bulging", etc. Within these applications, the robots run from 5 second to 6 second cycle times $^{3}$ and place products onto a pallet. Larry also discussed the differences between packaging, kitting, and palletizing from the system/customer, and SKU perspectives.

- Title: One Robot CAN Do It All

David Peters of Universal Robotics, Inc, as an integrator, was the only speaker who suggested that one robot can in fact do it all! David then went on to describe the philosophy behind the development of their product which is rooted in techniques from Artificial Intelligence. It uses sensor information to update the knowledge required for

\footnotetext{
${ }^{3}$ The Adept Cycle Time is defined as the time it takes a robot tool to perform continuous path, straight-line motions of $25 \mathrm{~mm}$ up, $305 \mathrm{~mm}$ over, $25 \mathrm{~mm}$ down, and back along the same path. Next Generation Packaging, Kitting, and Palletizing: Can one robot do it all?
} 
handling tasks across different applications such as random box moving, mixed palletizing, bin picking, and box picking. Universal Robotics develops device independent controls with their Autonomy product that supports collision avoidance and auto inverse kinematic determination.

- Title: Pick, Pack, Pallet: Can one robot do it all?

Erik Nieves of Yaskawa America, Inc., Motoman Robotics Division, presented a taxonomy of packaging robots and showed that kitting is a subset of bin picking. He proceeded to elaborate on how packing and palletizing are also subsets and that bin picking can be thought of as the superset from which the other three can be deduced. Erik argued that "one robot should not do it all" and in fact one robot should deal with going from unstructured to structured, one to create cases, and one to create pallets. He also stressed the need for a standard robot language and that the success of such a language depends on a ubiquitous and well defined vocabulary.

- Title: Exploiting Robotic Flexibility: Successes, Challenges, and Opportunities Dick Motley of Fanuc Robotics listed a series of successes in various application areas and his views on why and how the successes have been possible. He also described challenges and opportunities in these areas. In summary, he identified product proliferation, short product life cycle, small batch runs, regulatory influences, and labor issues as the areas that require attention and capital cost, change-over time, portability, and future flexibility as the perceived barriers.

- Title: Automated Robot Order Assembly

Christian Wurll of KUKA Systems North America discussed KUKA's experiences in terms of robotic applications for material handling and mixed case palletizing applications. In addition to kitting, packing, and palletizing, he noted yet another similar task that can be added to this list: assembly tasks. Christian argued that, in spite of similarities, one robot cannot do it all due to varying demands on payloads, reachability, packaging types, speeds, and component costs. He outlined how these similar tasks can be carried out by employing a modular software framework and provided several example scenarios where KUKA has had success using their AuROrA (Automated Robot Order Assembly) framework. In addition to modular software and control concepts, he also emphasized the importance of standardized communication interfaces, flexible planning approaches, and easy programming and configuration capabilities.

\section{Can one robot do it all?}

With the exception of one speaker, the consensus among the attendees was that one robot should not and cannot do it all. This is due to the differences in items such as payloads, reach, and grasping requirements. Based on discussions, we suggest the following definitions for kitting, packaging, and palletizing: 


\subsection{Definitions}

- Kitting is the process by which individually separate but related items are grouped, packaged, and supplied together as one unit. For example, automobile manufacturers typically make multiple versions of a vehicle on the same assembly line. Each vehicle may have different options, trim designs, etc. Frequently, these items are assembled into a kit and delivered to the assembly line for workers to integrate onto each individual vehicle.

- Packaging can be thought of as a set of processes (such as cleaning, drying, and preserving) and materials (such as glass, metal, paper or paperboard, plastic) employed to contain, handle, protect, and/or transport an article. The role of packaging is broadening and may include functions such as to attract attention, assist in promotion, provide machine identification (barcodes, etc.), impart essential or additional information, and help in utilization.

- Palletizing can be defined as the methodology of storing and transporting goods stacked on a pallet, and shipped as a unit load. It permits standardized ways of handling loads with common mechanical equipment such as fork-lift trucks.

\section{Next Steps and Future Considerations}

An open discussion was held towards the end of the seminar to discuss what areas can be targeted both from short-term and long-term perspectives. The hour-long discussion retouched on several points raised by the presenters and in the ensuing discussions. Below is a summary of the key points:

\subsection{Symbiotic role of Integrators and End-users}

The attendees stressed the need for seamless transition between robots and humans. One of the speakers suggested that a robot is the appropriate one for a particular task when that robot can be taken out of the line and a human can take over the robot-assigned task. Such seamless transition requires the system integrator to be aware of such requirements and be able to accommodate these needs. Towards this end, the attendees strongly felt that the robot system integrator community needs to grow to serve small and medium sized manufacturers. The robot integrator should also be able to combine disparate technologies to meet the needs of the end-user. Another suggestion that came up was to foster the idea of seeing the role of integrators as service providers who can empower the end-users who provide the necessary requirements to achieve desired solutions.

There was also wide agreement on coming up with a different business model for packing, picking, and palletizing tasks where the existing models do not hold. It was suggested that the thinking needs to change in terms of putting robots on the expense side of the ledger (like labor) instead of on the capital equipment side. The lack of robotics expertise of people who work and cooperate with the robots was also cited as an area requiring further training and corrective steps. 


\subsection{Standardization and Performance Metrics}

In general, the group lamented the fact that so many vendors neither use particular standards nor conform to existing best practices. Though it is a challenging task to come up with a common standard for various purposes, the group agreed on the following as a good initial set of questions for which the community (vendors, end-users, integrators, academia, and NIST) should seek answers:

- How can existing systems be evaluated and tested?

- What performance measures and test methods are appropriate?

- Can a common representation be developed for world, plan, and execution knowledge?

- How can we standardize control software across different platforms?

- Can we ensure PackML compliance across platforms?

- Can robots (end effector, conveyor, etc.) themselves be standardized?

- How can we reduce dependency on robot programming?

- How can standardized communication interfaces and flexible planning approaches be developed?

- Can we agree upon a common robot language and what are the steps that need to be taken? (One suggestion was to form a committee to address robot language standards).

In particular, the attendees emphasized the role NIST can play in the following areas:

- Development of a uniform representation (standard) of data associated with the products. For instance, can we match SKUs with characteristics of packaging wherein the SKU would contain the dimensions, etc.?

- Development of performance metrics for robot capability

- Development of standards for describing robot capability

- Development of performance metrics for end effectors and vision systems

- Quantification of the actuation time of end-effectors

- Development of generic process control

- Development of an open source protocol

A Google group titled nextgen_RobPKP (groups.google.com/group/nextgen_robpkp?hl=en) has been setup to facilitate discussions between the interested members of the community. To join or to post a message to the group, send an email to <nextgen_robpkp@googlegroups.com>. 